$\begin{array}{ll}\text { Italique } & \text { Italique } \\ \text { Poésie italienne de la Renaissance }\end{array}$

XVIII | 2015

Varia

\title{
La «bouche éloquente» di Porzia. Nuove proposte sulla Porzia (1664) di Elisabetta Sirani
}

Frédérique Dubard de Gaillarbois

\section{(2) OpenEdition}

\section{Edizione digitale}

URL: http://journals.openedition.org/italique/415

DOI: $10.4000 /$ italique.415

ISSN: 1663-4438

\section{Editore}

Librairie Droz

\section{Edizione cartacea}

Data di pubblicazione: 1 ottobre 2015

Paginazione: 151-186

ISBN: 978-2-600-01900-2

ISSN: 1423-3983

Notizia bibliografica digitale

Frédérique Dubard de Gaillarbois, «La «bouche éloquente» di Porzia. Nuove proposte sulla Porzia (1664) di Elisabetta Sirani », Italique [Online], XVIII | 2015, online dal 01 octobre 2017, consultato il 03 mai 2019. URL : http://journals.openedition.org/italique/415 ; DOI : 10.4000/italique.415

(C) Tous droits réservés 
Frédérique D Ubard DE GAILLARbois

L A «B O U C H E É L O Q U E T E»

D I P O R Z A.

NUOVE PROPOSTE

S ULLA PORZIA (1664)

D I E L I A B ETTA S I A N I

Intendami chi può, che m'intend'io Petrarca, Rvf IO5, $17^{\mathrm{I}}$ 


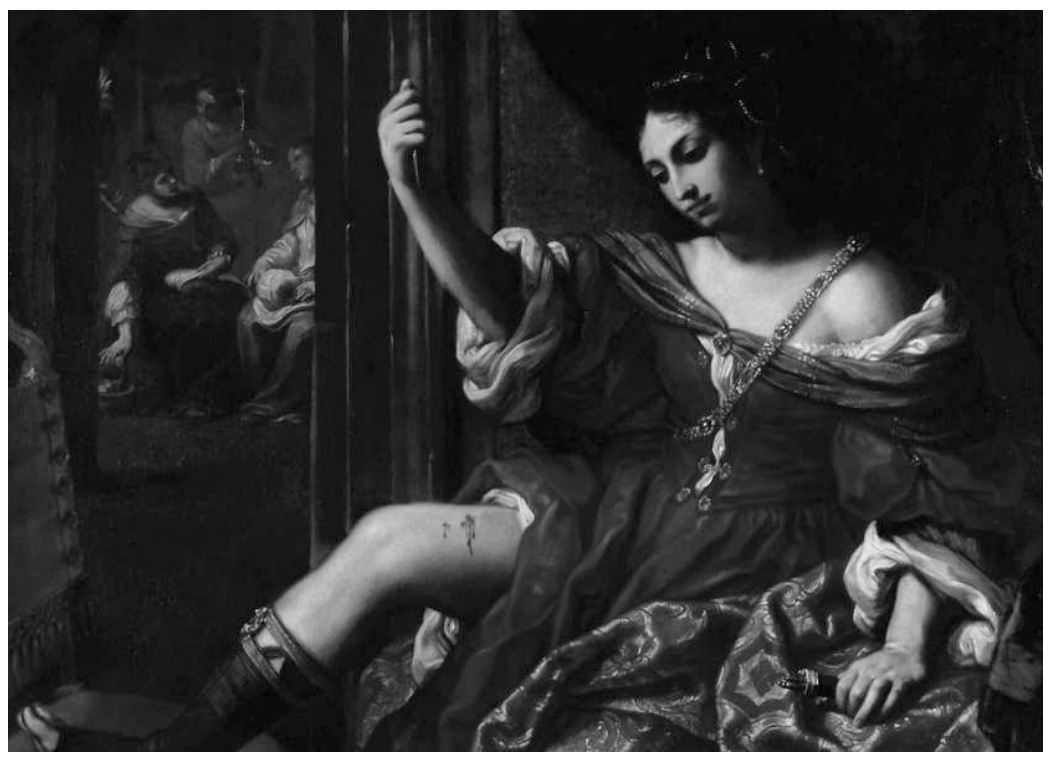




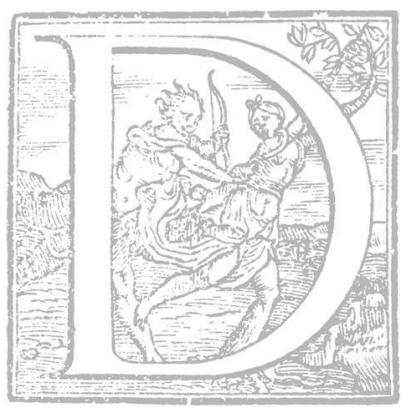

a quando è ricomparso negli anni 70, prima a Parigi, poi a New York, poi a Los Angeles, poi a Houston, per tornare finalmente a Bologna, dopo una sosta in occasione dell'importante mostra monografica allestitavi nel 2004, ${ }^{3}$ il quadro di Elisabetta Sirani è stato visto, riprodotto, diffuso. Non per questo, e nonostante l'indubbio fascino da esso esercitato, ci sembra sia stato ancora oggetto di uno studio né approfondito né specifico. ${ }^{4}$

\section{BILANCIO INIZIALE}

Se le peripezie commerciali del quadro meriterebbero di essere ricostruite fin dall'inizio, la parentesi americana potrebbe corrispondere ad una riscoperta ma anche ad una percezione, fortemente connotata ideologicamente della Porzia siraniana in chiave prima femminista, ${ }^{5}$ poi, improntata ai "Women Studies". Pietra miliare da quelpunto di vista fu la mostra allestita a Los Angeles nel $1976 .^{6}$

Se tale angolatura è indubbiamente servita ad esumare e svecchiare il quadro, le analisi fatte all'epoca appaiono a loro volta datate, in parte discutibili e soprattutto parziali. Vanno integrate con le altre chiavi di lettura disponibili, onde ricostruire un approccio idealmente a tutto tondo di un quadro dalla notevole complessità semantica, dal momento che la Porzia siraniana potrebbe chiamare a consulto non solo i lumi e le perizie degli storici dell' arte, ma degli studiosi della letteratura antica e moderna in quanto pittura di storia, strettamente dipendente da una fonte letteraria. Inoltre la filosofia, la politica, la psicologia ma anche la storia delle arti minori potrebbero dare un contributo nello sciogliere $i$ diversi enigmi di un quadro cosicomplesso.

Contributo precipuo della mostra monografica del 2004 fu di strappare la figura di Elisabetta Sirani alla doppia paternità siraniana e reniana, di collocarla rispetto ai colleghi (più che ai padri, veri o presunti), di mettere a fuoco un'identità ed una traiettoria artistica, dando particolare risalto alla polivalenza tecnico-artistica (disegni, stampe, acquerelli), all'attività didattica ${ }^{7}$ finora sconosciuta e alla consapevolez?a artistica. ${ }^{8}$

Altro merito di questa mostra, l'aver tenuto dissociate l'artista dalla donna; una confusione non solo fatale per le donne-artiste, ma insita nelle 
prime fonti disponibili su Elisabetta Sirani. La fortuna in senso lato della Sirani rimane succube di questo intreccio. La mostra ha cercato invece di focalizzarsi sul lavoro e le opere della Sirani, a prescindere dalle leggende negative epositive.

\section{UN'INEDITO ICONOGRAFICO?}

È d'uopo ricordare che senza la rubrica della Nota delle pitture fatte da me trascritta da Carlo Cesare Malvasia nella biografia «Di Giovanni Andrea Sirani e di Elisabetta sua figliola» che chindeva la Felsina pittrice (I678), ${ }^{9}$ la Porzia brillerebbe per la sua assenza, pure nella letteratura critica coeva e comunque in quelli che possiamo considerare gli incunaboli della critica siraniana: ovvero, la biografia malvasiana della Felsina pittrice e le due raccolte di Picinardi, ${ }^{\mathrm{IO}}$ florilegi di poesie scritte da un'ottantina di pennaioli in occasione sia della consegna o esposizione di diversi quadri siraniani che della morte della pittrice; materiale di prima mano sulla ricezione e sul tessuto relazionale della Sirani, finora pochissimosfruttato.

Anche se diverse poesie vennero scritte viva la pittrice, le raccolte furono pubblicate postume. L'unica a parlare della Porzia fu quindi la stessa Elisabetta, la cui sintetica descrizione:

Una Porzia in atto di ferirsi una coscia, quando desiderava saper la congiura che tramava il marito; quadro soprauscio, e di lontano in un'altra camera donzelle che lavorano, per il sig. Simone Tassi ${ }^{11}$

può essere confrontata alle altre due attestazioni coeve disponibili, entrambe di Malvasia: una nell'Indice della Felsina da lui stilato, strumento di lavoro notevole, sebbene non neutrale, come abbiamo avuto occasione di rilevare altrove: ${ }^{\mathrm{I2}}$

Porzia [...] della Sirana, e che si dà ferita nelle coscia, per provarsi valevole a tener segreta la congiura. ${ }^{\mathrm{I}}$

L'altra negli appunti inediti dello stesso ove il quadro è oggetto di un aggiornamento riguardante cambi di proprietà:

Del i67I, Luglio, il Foschi merciaro comprò da' Bordani, nell'eredità di Simon Tassi, la Porzia che si avvezzi alle ferite col percuotersi una coscia 
con un feratore, sovrauscio, mezza figura della Sirana, ed Absalone ch'è appesa della sua chioma per venderla a cento dinari, di mano del Sirani compagno del sù detto quadro, lire 725 , stimato 600 prima. ${ }^{14}$

Insomma le tre menzioni della Porzia sono o di Malvasia o trascritte da Malvasia. Queste tre descrizioni, per quanto spicce, presentano sottili varianti sul movente di Porzia. Anche il termine «feratore» andrebbe indagato. Nel mentovare il cambio di proprietà, lo storico evoca en passant il differenziale di valore tra i due soprausci, giacchè la Porzia di Elisabetta faceva il paio con una Absalon (che sarebbe una Semiramide) del padre. Ora, nel I67I, risulta che il quadro della figlia valesse ormai molto di più di quello del padre; informazione perfettamente in linea col succo degli appunti, ove Malvasia attribuisce alla figlia una superiorità artistica (ma anche umana) taciuta o non dicibile nella Felsina.

A spiegare il silenzio dell'entourage siraniano e malvasiano, potrebbe essere il destinatario del quadro: Simone Tassi, un ricco mercante, un esponente di questa borghesia agiata, che costituisce una porzione tanto meno irrilevante della clientela siraniana che stando a W. Chatwicke, ${ }^{, 5}$ avrebbe commissionato o acquistato le opere più originali della Sirani; opere che fanno colpo oggi, mentre sarebbero passate inosservate all'epoca.

La considerazione di W. Chadwick sembra valere appieno per Porzia, di cui ci si meraviglia che non abbia «sfondato», mentre quadri precedenti ed affini per certi versi avevano riscosso successo ed ottenuto omaggi poetici. Si pensi in particolare alle altre donne forti della Sirani: Dalila (I657), Giuditta (I658), Timoclea (I659), e sopratutto Iole (I662). Non solo questi quadri riscossero omaggi poetici ma la Sirani venne identificata con queste figure, come se questi ritratti venissero 'pacificamente' letti come autoritratti.

Una quarta descrizione ci viene fornita dalla trascrizione accurata e preziosa degli inventari di alcuni committenti e collezionisti bolognesi stilata da Raffaella Morselli, tra cui quello del Tassi, per l'appunto. Tale lavoro consente di collocare la Porzia in un insieme, di vedere come, dove e con chi il quadro venne disposto - accostata quasi sistematicamente ad una tela paterna onde consentire un raffronto, chiaramente apprezzato non solo dalla critica, ma dagli stessi clienti - ma anche come veniva qualificato: 


\section{La moglie di Bruto opera della Sirana con cornice DORATA L 500. ${ }^{\text {I6 }}$}

La dicitura è interessante in quanto Porzia è 'tornata' moglie di Bruto al punto che il nome della donna scompare dall'inventario. Allo stilatore dell'inventario premeva invece sottolineare la presenza di una cornice e il valore globale dell'opera. La dimensione economica elusa nella Nota della pittrice campeggia, quindi, nell'inventario del comittente. Perchè mai quindi la Porzia passò inosservata? Non si può imputarne con certezza la colpa a Simone Tassi, 'reo' di non essere stato un aristocratico colto, un accademico "gelato", ma soltanto un ricco mercante. Non per questo, acquirente casuale, se possessore di quadri sia della Sirani che del padre (e del Guercino). Non si sa per altro se Tassi avesse commissionato il quadro o l'avesse acquistato già fatto. Quel Simone Tassi possedeva altre due pitture di storia antica: una Venere, una Geometria e una Sibilla, tre quadri sacri della Sirani (due Madonne). Uno di essi, la pala di Sant'Antonio, suscitò qualche omaggio poetico, il che ci induce a pensare che non fu tanto il committente a non favorire un'esposizione ${ }^{17}$ pubblica del quadro di Porzia quanto un impedimento legato al quadro stesso, il cui soggetto profano, la cui arditezza formale, per l'appunto, l'avrebbe reso quasi invisibile? Ma non è neppure il fatto che il quadro sia stato profano ad essere dirimente, se si pensi al successo riscosso dal un Ercole filante, un altro quadro perso o scomparso, attestato da un'imponente produzione poetica per quanto scherzosa. ${ }^{18}$ Non si può negare che la Sirani abbia giocato con l'inversione delle parti tra maschile e femminile in almeno quattro quadri - Dalila (I657), Timoclea (I659), Ercole come pendant di una Iole (I662), Porzia (I664) - ma è come se in quest'ultima avesse passé les bornes; limiti ancora avvertibili se il quadro, per quanto affascinante, suscita disagio e sembra opporre una tenace resistenza alle pretese ermeneutiche.

Non potrebbe essere che la sfortuna critica della Porzia tradisca un imbarazzo di fronte ad un'opera decisamente spiazzante, di non agevole lettura nella Bologna del 600? Che i motivi per cui la Porzia piace cosi tanto al giorno d'oggi, al punto da apparire come il capolavoro di Elisabetta Siriani siano quelli stessi per cui non sarebbe invece piaciuta nel 600? Non potrebbe essere che la Porzia «sia sfuggita» alla Sirani, 
secondo una felice espressione di Vera Fortunati? Non è che l'originalità venisse ricercata a quei tempi; $i$ pittori si cimentavano tutti negli stessi soggetti; proponevano variazioni su temi noti, gareggiando più formalmente che contenutisticamente tra di loro. Ora, la Sirani con la sua Porzia automutilante avrebbe commesso una specie di hapax; ${ }^{19}$ un inedito iconografico.

Tra le donne forti, sia pur di grande successo nell'iconografia seicentesca, Porzia non è certamente fra le più comuni. Inoltre, l'immagine più diffusa della Porzia era non già quella dell'automutilazione, bensi del truculento suicidio compiuto nell'inghiottire carboni ardenti. Se gli esempi non abbondano, si trovano. ${ }^{20}$ Stupisce in particolare che non sia stata riscostruita una possibile trafila bolognese, giacchè l'utilissimo Indice della Felsina, stilato dal Conte Malvasia, registra una Porzia reniana $^{2 \mathrm{I}}$ nonchè savonanziana, ${ }^{22}$ cui andrebbe aggiunta un'altra Porzia automutilante, attribuita ad Alessandro Tiarini, ${ }^{23}$ «il Seneca de' pittorì, stando a Malvasia. Ma sarà meglio lasciare queste investigazioni agli storici dell'arte, bolognesi e non, e volgerci alla fortuna letterararia seicentesca di Porzia, foriera, come tenteremo di dimostrare, di acute letture del gesto di Porzia.

\section{LE STORIE DELLA PITTURA}

Trattandosi di una «pittura di storia» converrà iniziare con uno studio riavvicinato del passo plutarchiano che sta a monte del quadro ed esplicitare implicazioni forzatamente elittiche. La ricostruzione della fortuna letteraria di Porzia $i$ cui tasselli principali sono Plutarco, Valerio Massimo, Boccaccio ci consentirà di evidenziare la discontinuità tra Plutarco e gli altri e per la lunghezza e ricchezza del contenuto, di sottolineare la differenza tra una Porzia quattro e cinquecentesca sostanzialmente «censurata» e smussata negli angoli più spigolosi e ridotta ad exemplum non problematico di fedeltà coniugale sulla linea moralistica di Valerio Massimo e la riscoperta seicentesca della Porzia plutarchiana, di cui la Porzia siraniana offre una clamorosa ed eccezionale testimonianza in ambito iconografico.

Tenteremo poi di dimostrare come a monte del quadro della Sirani ci siano non uno ma due passi della Vita di Bruto, il che ci consentirà di proporre una lettura inedita della contrapposizione tra il primo piano (individuale) e il secondo (collettivo) del quadro. 
Lo spettatore è subito colpito dal contrapposizione tra il gesto solipsistico, aristocratico, abnorme di Porzia in primo piano e l'azione, invece, collettiva, tradizionale, conforme del quartetto inteso a cucire. Si distinguono fuso, rocca, tomboli, una cesta di lavoro; contrapposizione che doveva pur stare a cuore a Elisabetta Sirani, se richiamò proprio l'attenzione su di essa nella rubrica della Nota delle pitture fatte da me dedicata a Porzia:

Una Porzia in atto di ferirsi una coscia, quando desiderava saper la congiura che tramava il marito; quadro soprauscio, e di lontano in un'altra camera donzelle che lavorano, per il sig. Simone Tassi. ${ }^{24}$

Verrebbe da contrapporre un quadretto di genere fiammingo, raffigurante una «scuola di femmine», un interno, una scena insieme domestica e lavorativa al ritratto eroico-tragico-storico di Porzia. Il quartetto partecipa della tradizione, Porzia della Storia.

Non sarà però la letteratura italiana coeva a consegnarci le chiavi ermeneutiche di questa contrapposizione bensi quella francese ove Porzia compare non solo in tragedie (Garnier, Guerin de Bouscal, Boyer) ${ }^{25}$ ma nella trattatistica (Du Bosc, Scudéry, Lemoyne) dell'epoca e dove qualcuno si sofferma anche a lungo, sul gesto dell'automutilazione.

E poco verosimile che l'Italia debba aspettare l'Alfieri per far comparire per inciso e di riflesso una Porzia auto mutilante ${ }^{26}$ nel suo teatro? Stupisce che il sensazionalismo barocco non abbia gradito questa scena dell'orrore coningale. Se le tragedie francesi sfruttano il potenziale politico di Porzia magari al servizio di cause opposte, ${ }^{27}$ la trattatistica francese la inserisce in un disegno filogino ${ }^{28}$ ambizioso ed articolato, ove sia l'automutilazione che il suicidio vengono accuratamente commentati. Non solo Bruto fa da faire-valoir a Porzia di cui viene proclamata e spietatamente disquisita la superiorit $\hat{a}^{29}$ ma gli autori francesi risultano sensibili alla portata politica ${ }^{30}$ e filosofica ${ }^{31}$ dei gesti di Porzia che appare totalmente rimossa dalle rivisitazioni italiane moralizzanti.

Il suicidio venne normalizzato in prova estrema di fedeltà coniugale nella letteratura italiana, mentre si prestava a letture in chiave politica e filosofica, ${ }^{32}$ di cui abbiamo trovato, però, esempi solo francesi. La parola «stoica» è il leitmotiv di questa lettura filosofica ${ }^{33}$ dei gesti di Porzia che ne farebbe una «amazzone cristiana», un'icona dello «stoicismo cristiano» in voga nel '6oo. 
Il confronto tra la ricezione francese ${ }^{34}$ e italiana di questa femme forte conferma quanto isolata e straordinaria sia la Porzia siraniana nell'ambito italiano, sia dal punto di vista iconografico che letterario, il che potrebbe spiegare la scarsa fortuna critica di cui ha fatto le spese.

\section{LA FONTE PLUTARCHIANA}

La fonte letteraria del quadro è stata identificata da tempo. Contributo fondamentale fu l'articolo di S. Sabbatini che consenti di accertare la presenza delle Vite plutarchiane nella biblioteca dei Sirani grazie al ritrovamento di un'inventario dei beni stilato nel I666 e in particolare dei libri lasciati da Giovan Andrea ad Anna Maria Sirani, sorella più giovane di Elisabetta. ${ }^{35}$

L'originalità del quadro, la sua innegabile dipendenza dalla fonte plutarchiana, nonché la presenza accertata delle Vite plutarchiane nella biblioteca siraniana inducono a pensare che fu Elisabetta a scegliere il soggetto, a ritagliare l'episodio dell'automutilazione di Porzia nel tessuto di questa vita lunga, per altro, ove la donna compariva a cinque riprese. Di una comparsa, Elisabetta Sirani fa una protagonista. In quanto a Bruto, soggetto principale della biografia plutarchiana, viene letteralmente espulso ${ }^{36}$ dal quadro. Mutatis mutandis si potrebbe osservare che la sorte subita da Bruto nel quadro della Sirani è la stessa di Giovan Andrea nella biografia di Malvasia.

Andrebbe indagata la presenza di Plutarco nella produzione siraniana e non, coeva.

Stando alla Nota della Sirani, almeno quattro suoi quadri proverrebbero dalle Vite parallele: uno dalla Vita di Bruto per l'appunto, e tre da quella di Alessandro. Si suole parlare solo di Timoclea, per le affinità tematiche con la Porzia, ma la Nota registra altre due pitture di storia plutarchiana tratte dalla Vita di Alessandro, passate inosservate, o perché scomparse, o perché non rientranti nell'ottica 'femminista. ${ }^{37}$

Non si può non citare in extenso il passo cui la Sirani avrà attinto, nella traduzione italiana cinquecentesca di Ludovico Domenichi (pubblicata nel I555, ma riedita nel 1607 e 1620, stando a S. Sabbatini): ${ }^{3}$

Ma Bruto, si come quegli, che si era fatto capo, e guida d'una tanta impresa a' primi cittadini di Roma per grandezza d'animo, per nobiltà, e 
per valore, seco medesimo pensando, e considerando ogni pericolo, che poteva avvenire, e dimorando nelle difficultà di questa cosa, non poté stare ascoso alla moglie, che dormiva seco; percioch'ella lo vedeva tutto pieno di pensieri, e di travagli contra il suo costume, e ch'egli macchinava fra se medesimo un disegno di grande importanza, e da non isciorsi così tosto. Questa era Porcia, come io dissi di sopra, figliuola di Catone; la quale Bruto haveva havuta per moglie da Catone suo zio, non fanciulla, ma vedova, che gia l'era morto il primo marito; il quale l'haveva presa fanciulla, e di lei havea havuto un bambino, c'havea nome Bibulo. Leggesi ancora hoggi una certa operetta di questo Bibulo, de'detti, e fatti di Bruto. Ora essendo Porcia donna molto savia, \& volendo bene al marito, \& oltra ciò dotata d'animo grande, e prudente, non prima hebbe ardimento domandare al marito i segreti del suo cuore, ch'elle havesse fatta questa esperienza di se stessa. Perche pigliando un picciolo coltello, col quale i barbieri sogliono tagliar l'unghie, e cacciando di camera tutte le sue cameriere, si fece une gran ferita in una coscia, onde uscì di molto sangue: e e di là a poco quella ferita le mise addosso un grave dolore, \& una terribil febre. Della qual cosa attristandosi Bruto, e molto increscendogliene, allhora, che il dolore piu la strigneva, gli arcionò in questo modo. «Io, o Bruto, figliuola di Catone fui messa in casa tua, non come bagascia, perche io partecipassi solamente del letto, e della tavola, ma accioche io havessi parte teco delle cose liete, e delle triste anchora. Quanto appartienne a te, veramente in questo nostro matrimonio non è cosa alcuna che meriti riprensione; ma io con che segnale ti dimostrerò l'animo mio, e come potrò ristorarti, se anchora io non sopporto la tua passione, \& non fossero i tuoi pensieri degni di fede? Io so, che la natura delle donne è fragile a ritenere i segreti, ma io, o Bruto mio, hò in me una certa forza e di buona creanza, e d'ottima consuetudine oltra lo ingegno naturale; e mi conosco essere figliuola di Catone, e moglie di Bruto. Nelle quai cose fidandomi io prima poco, hora hò conosciuto per pruova, ch'io non mi lascierei vincere dal dolore.» Dette queste parole gli mostrò la ferita, e gli scoperse la pruova, ch'elle haveva fatta di se medesima. Allhora Bruto spaventato \& alzando le mani al cielo, pregò gli Dei, che riuscendogli valorosamente i suoi disegni, lo facessero riputare marito degno di Porcia: e poi amorevolmente confortò la moglie. ${ }^{39}$

Questo passo straordinario meriterebbe un commento a sé stante. Tra sfida e cautela, rivendicazione di parità e abile omaggio all'auctoritas paterna e maritale, Porzia dimostra tatto, sangue freddo, ammirevole capacità argomentativa. 
Tale eloquenza viene però ridotta ad ekfrasis: chiosa secondaria, nel senso proprio e traslato del termine, del gesto cui ha preferito affidare il proprio messaggio. Il primato dato da Porzia al gesto sulla parola è proprio quanto preme alla Sirani che, non a caso, e contrariamente ai pochi emuli, che siano predecessori o epigoni, hanno, tutti, raffigurato il versante 'locutorio' dell'episodio che consentiva tra l'altro di richiamare Bruto e di «coningalizzare» Porzia.

Rispetto al gesto tremendo ed equivoco, la parola svolge un ruolo razionalizzante, eufemizzante, conciliativo. Fanno specie però la facilità con cui Porzia è riuscita a indovinare $i$ segreti di stato del marito inferiti dai soli «disturbi del sonno» e l'inettitudine maritale che non solo non anticipa ma non capisce alcunché. Bruto risulta indubbiamente sminuito - volendo, castrato - dalla palese, dimostrata e da lui stesso riconosciuta superiorità della moglie.

A noi premerà sottolineare l'inversione delle parti tra gesto e parola nella fonte letteraria e nella pittura di storia; ovvero, mentre nel testo plutarchiano, il gesto precede la parola: la lezione, «l'arcione» di Porzia, nel quadro è la parola scritta, il testo plutarchiano, la storia a precedere l'immagine.

Il fatto che la Sirani abbia scelto di rappresentare il fare e non già il dire di Porzia, contrariamente ad esempio sia ad Ercole de' Roberti che ad Alessandro Tiarini e volendo anche a Francesco Solimena o Bernard Lepicié (per fare esempi posteriori ed esotici) è cruciale. Non solo de' Roberti, Tiarini, Solimena e Lepicié hanno raffigurato la coppia e quindi il dopo, la fase posteriore all'azione: quella retorico-didascalica in cui Porzia «spiega» a Bruto e giustifica il gesto. Non solo, quindi, la Sirani ha fatto la scelta caravaggesca (guerciniana?) non del dopo (come nella sua Giuditta I658), ma del durante (come nella Timoclea), ma è l'unica ad aver filologicamente rispettato il testo plutarchiano, ovvero ad esibire una coscia, mentre tutti gli altri (tranne Lepiciè), compresa la settecentesca riscrittura alfierana ${ }^{40}$ del passo, hanno spostato la ferita dalla coscia in giù o in su.

Non è solo una logica estetica, la scelta dell'orrido, dell'effetto a dettare questa scelta. Cosi come Porzia punta prima sullimmagine, poi sul 'verbo', la Sirani propone con Porzia un quadro metacritico che altro non sarebbe che un elogio dell'espressività gestuale, una illustrazione magistralmente figurativa dell'impatto comunicativo dell'immagine rispetto alla debolezza della parola. 
Il quadro sarebbe doppiamente agonistico in quanto celebrerebbe sia la superiorità di una donna sull'uomo che la superiorità della pictura sulla poesis, disputa insieme annosa e vitale, universale e locale; arena nella quale la Sirani scenderebbe magistralmente con la sua Porzia, nel farne più un modello di eloquenza che di amore coniugale; un'eloquenza strettamente identificata con quella pittoresca.

\section{Controesempio}

Per apprezzare il grado di rottura compiuto dalla Sirani nella sua lettura di Porzia, sarà d'uopo ricordare la doxa preesistente. Nella letteratura italiana l'immagine predominante di Porzia era quella edificante ma minimalista consegnata da Valerio Massimo, che l'aveva ridotta ad exemplum di «amore coniugale». Valerio Massimo si soffermava esclusivamente sul suicidio di Porzia, sottolineandone per altro il carattere imitativo rispetto a quello paterno, spostando la questione del confronto, non più tra marito e moglie, ma tra figlia e padre, e riconoscendo a Porzia un'inventività puramente strumentale:

Tuos quoque castissimos ignes, Porcia M. Catonis filia, cuncta saecula debita admiratione prosequentur. Quae, cum apud Philippos uictum et interemptum uirum tuum Brutum cognosses, quia ferrum non dabatur, ardentes ore carbones haurire non dubitasti, muliebri spiritu uirilem patris exitum imitata, sed nescio an hoc fortius, quod ille usitato, tu nouo genere mortis absumpta es. ${ }^{41}$

Non il suicidio ma il modo, in quanto novo sarebbe stato superiore a quello usitato, anche se Valerio Massimo non se la sentiva di affermarlo categoricamente ( $(s e d$ nescio an hoc fortius»), pedissequamente seguito in materia da Boccaccio.

Basterebbero queste considerazioni, anche sommarie, a far misurare l'atipicità della Porzia siraniana che non è suicida bensi automutilante e che più che esempio di fedeltà e di dedizione coniugale sembra mettere in crisi e in questione un marito, destinatario di una tremenda e insolita rivendicazione.

La Porzia di Elisabetta non è quella di Valerio Massimo ${ }^{42}$ che aveva soppresso l'episodio dell'automutilazione. Se la microbiografia di Boccaccio è considerevolmente più dettagliata della vignetta di Valerio Mas- 
simo e non censura l'automutilazione, presenta varianti cosi considerevoli rispetto alla fonte plutarchiana da far pensare o che abbia attinto ad altre fonti di cui avrebbe fatto una sintesi o che abbia riscritto liberamente il copione plutarchiano.

Qui integritatis Portie conscius, cum eidem scelesti facinoris aperuisset archanum, factum est ut, ea nocte cui dies illuxit qua Cesar humanis rebus coniuratorum opere subtractus est, exeunte Bruto cubiculum, Portia tonsoris novaculam summeret, quasi unguium superflua resectura; fingensque illam sibi caso cecidisse, se ex animo vulneravit. Sane astantes ancillule cum manare vidissent sanguinem, suspicate aliquid gravius, clamore sublato, abiens Brutus in cubiculum revocatus est et ab eo obiurgata Portia quoniam tonsoris precipuisset officium. Portia autem, semotis ancillulis, inquit: «Minime temerarie factum quod putas est; tentatura autem quo animo me ipsam gladio perimere et mortem perpeti possem, si minus tibi pro votis cepta succederent, feci». ${ }^{43}$

Il resoconto boccaccesco dell'episodio varia sia nei tempi che nello svolgimento che nell'interpretazione del gesto compiuto da Porzia. Nella vita boccacciana, Bruto non viene scoperto dalla moglie ma le rivela spontaneamente il segreto della congiura. Porzia compie il gesto l'indomani della congiura mentre in Plutarco non si precisa quando, ma l'impressione è che si svolga prima di essa. Mentre nel testo plutarchiano non si parla delle ancelle se non per dire che siano state cacciate, sono invece presenti nella versione boccacciana. Il malinteso di Bruto che scambia la ferita di Porzia per un infortunio cosmetico, sgridandola addirittura, tocco umoristico del Boccaccio, è del tutto assente dal testo plutarchiano, ove Bruto risulta prima esterrefatto poi ammirato. Last but not least, il movente addotto dalla Porzia boccacciana, a prescindere della laconicità rispetto alla «concione» plutarchiana, non è una richiesta di compartecipazione, ma una dimostrazione della capacità a suicidarsi. Importante contributo, però, (dal nostro punto di vista) della biografia boccacciana ${ }^{44}$ è la non censura dell'automutilazione e il suo esplicito collegamento col suicidio venturo, che diventerà la chiave di lettura dominante. Tale collegamento non era stato fatto da Plutarco:

Quod cum audisset Portia nil sibi, subtracto viro, letum futurum existimans, arbitrata non alio animo mortem passuram quam olim cultri tonsorii vulnus, confestim in vetus propositum venit; et cum ad voluntariam 
necem nullum sibi adeo cito, ut impetus exposcebat, prestari videbatur instrumentum, ignitos carbones, quos forte propinquos habebat, indubitanter manibus gucturi iniectos exhauxit; a quibus precordia exurentibus spiritus vitalis abire in mortem coactus est. ${ }^{45}$

Boccaccio serba l'episodio dell'automutilazione ma lo banalizza e normalizza riducendolo a brutta copia, a prova del suicidio finale.

Nella trattatistica filogina quattro e cinquecentesca, ove compare il nome di Porzia, non è la Porzia plutarchiana e men che mai quella dell'automutilazione che viene ricordata, bensi quel paradigma semplificato e normalizzato di fedeltà coniugale, la morte di Porzia essendo sentimentalmente assunta come rifiuto di sopravvivere al marito, una fedeltà estrema, alla Ipsicratea o Artemisia, cui Porzia verrà assimilata nei cataloghi di donne illustri. Mentre le possibili implicazioni politiche e filosofiche del gesto venivano occultate da questa piega sentimentale, il suicidio veniva riscattato dal movente coniugale. Si potrebbero fare fior di esempi da Sabadino degli Arienti ${ }^{46}$ a Galeazzo Flavio Capella ${ }^{47}$ ove Porzia compare in elenchi di donne illustri nella rubrica della fedeltà coningale.

Più interessanti potrebbero risultare le evocazioni di Porzia come modello suicida. L'abbiamo indentificata come tale nell'elegia di Madonna Fiammetta ${ }^{48}$ abbandonata dall'amante Panfilo e disperata al punto di contemplare, nonché nelle rime di una Vittoria Colonna appena vedova. Fiammetta è interessata ad una rassegna dei diversi modi di suicidarsi tra cui $i$ carboni di Porzia. Si focalizza sul modo più che sul movente o fine che potrebbe o riscattarlo o smussarne lo scandalo; scelta assai ardita.

La potenziale problematica etica - liceità del suicidio dal punto di vista religioso - viene invece affrontata in rime notevoli di Vittoria Colonna, disperata dalla morte de suo «Sole», Fernando de Avalos. Dopo aver evocato diverse figure di femminilità addolorata, luttuosa, addirittura rabbiosa e vendicativa (Penelope, Laudamia, Arianna, Medea), le viene in mente il nome di Porzia con cui sente maggiori affinità:

Porzia sopra ad ogni altra mi rivolse Tanto al suo danno, che sovente insieme Piansi l'acerbo martir nostro uguale.

Ma se breve ora forse ella si dolse,

Quant' io sempre mi doglio, poca speme 
D'altra vita miglior le diede altr' ale; E' 'l mio grave dolor vivo e immortale Siede nel core, e dell'alma serena

Vita immortal questa speranza toglie

Forza all' ardite voglie;

Nè pur questo timor d'eterna pena,

Ma d' ir lunge al mio Sol la man raffrena. ${ }^{49}$

A differenziare le due donne ad onta del comune lutto (reale per la Colonna, simbolico per la Fiammetta) e della tentazione suicida, è la «poca speme / D'altra vita miglior» che diede alla pagana «altr'ale» da chi viene raffrenata non già dal "timor d'eterna pena» quanto dal «ir lunge al mio Sol» con un atto irreversibile che le impedirebbe di ritrovarlo in paradiso.

L'angolatura e l'intonazione (anche per via della scrittura autobiografica, vera o finta che sia) di Fiammetta e Vittoria sono assai diverse rispetto alla doxa filogina. Il suicidio non appare più come riprova di fedeltà coniugale. Anzi, nel caso di Vittoria, la separerebbe dall'amato per sempre. Ciò non toglie che entrambe le donne disperate siano tentate da questa soluzione estrema e che Porzia appaia loro come un modello possibile.

Verrebbe da chiedersi perchè Porzia non riscosse la stessa fortuna iconografica, letteraria, politologica e teologica di una Lucrezia: eticamente ammirabile ma religiosamente condannabile.

L'automutilazione poteva anche ricollegarsi a modelli di auto-castighi molto diffusi nell'iconografia sacra, addirittura di moda nella Bologna seicentesca ove i san Girolami nel deserto e le Maddalene pentite abbondano nel cerchio dei Sirani e non. L'iconografia sacra era rigogliosa di piaghe esibite, di carne martoriata, di mutilazioni e scortificazioni inferte certamente più dagli altri che da se stessi. Sincretica era la cultura dell'epoca e sincretico sarà stato sia lo sguardo della pittrice che del pubblico. La normalizzazione del gesto di Porzia implicava due operazioni: nel primo caso allineare l'automutilazione sul suicidio, nel secondo puntare sulle forme (affini) più che sul contenuto (dissimile). $L$ 'associazione anche inconsapevole tra questi modelli familiari e il gesto inconsueto di Porzia non è da escludere.

La compatibilità tra una lettura cristiana e pagana dei gesti (prima automutilante, poi, suicida) poteva fare di Porzia una «amazzone 
cristiana»; un ossimoro ideologico che non ha nulla di eccezionale. ${ }^{50}$ Visto che gran parte della produzione della Sirani era sacra e che la pittrice viveva nella seconda città degli Stati della Chiesa, non è il caso di leggere la produzione profana in chiave anti-religiosa. Del resto, due degli autori francesi che più si soffermano su Porzia: Du Bosc e Lemoyne, erano, l'uno «cordelien», l'altro gesuita.

Inoltre, la produzione della Sirani annovera tre quadri in cui un soggetto si ferisce: la Porzia, una Maddalena autoflagellante (di Besançon), una raffigurazione allegorica dell' amor proprio ${ }^{5}$ in cui un angelo si ferisce da sé. La voce della Nota dedicata a quest'ultima opera comprende una citazione di un verso del Petrarca e poi dell'Ariosto: "Intendami chi può, che m'intendo io» che è passata inosservata, ${ }^{52}$ mentre potrebbe esssere ricca d'implicazioni anche sulla cultura letteraria della pittrice che non avra ignorato il Canzoniere $o$ l'Orlando furioso. ${ }^{33}$ É ragionevole pensare che il Petrarca $e$ l'Orlando furioso facessero parte della biblioteca e della cultura dei Sirani. ${ }^{54}$

Ultimo esempio, noto, ma giustificato da parametri locali e cronologici: La gloria delle donne (I690) di Giulio Cesare Croce; autore bolognese molto popolare, che non poteva essere ignoto ai Sirani. Egli inserisce banalmente Porzia in una serie di donne illustri ed esemplari che vanno contrapposte agli esempi di donne malvage e perverse addotte da autori misogni:

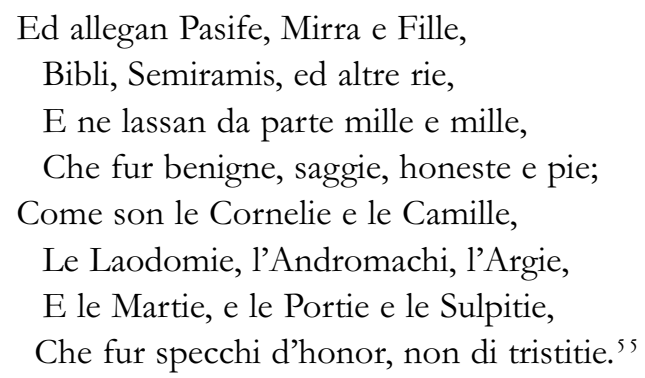

Da questo campionario parziale si può desumere che Porzia fosse diventata una figura scontata, quasi proverbiale nella letteratura filogina pre-seicentesca, addotta senza se e senza ma, purchè venissero rimosse le parti oscure o problematiche del personaggio.

La Porzia siraniana - automutilante - appare pertanto indissolubilmente legata alla volgarizzazione delle Vite parallele, nonché alla voga 
seicentesca di Plutarco. ${ }^{56}$ E chiaro che la Sirani si rifaccia ad una fonte in controtendenza rispetto alla «unlgata» su Porzia: una fonte alternativa: quella plutarchiana in quanto era l'unica a valorizzare l'episodio dell'automutilazione, l'unica ad entrare nei particolari. Rispetto a questa fonte, gli altri ritratti di Porzia appaiono vignette ad usum Delphini, immaginette d'Épinal.

La Porzia suicida era evidentemente molto meno problematica della Porzia automutilante a giudicare dalla fortuna sia iconografica che letteraria delle due. ${ }^{57}$ Se ne potrebbe inferire che la Porzia automutilante ne passe pas. Potrebbe essere che l'automutilazione sia più tabù del suicidio, con cui intrattiene pur una verosimile o rassicurante (almeno intellettualmente) parentela, com'aveva intuito Boccaccio. ${ }^{58} \mathrm{Gli}$ autori francesi, da Du Bosc agli Scudery, ${ }^{59}$ sottolinereanno ed espliciteranno questo collegamento.

Lo sdoppiamento di Porzia o Le DUe «STORIE» Della PITTURA

Porzia compariva in diversi momenti della plutarchiana Vita di Bruto: c'era la Porzia dell'automutilazione prima della congiura (XV); la Porzia prima agitata al punto dall'essere paragonata ad una baccante, poi svenuta (XVIII); la Porzia disperata quando Bruto dopo l'uccisione di Cesare deve esiliarsi in Lucania (XXVII); la Porzia suicida (LXI). I quattro episodi si possono illuminare ma anche contraddire a vicenda. Se il suicidio finale ha spesso chiarito retrospettivamente l'automutilazione, la partenza di Bruto rivela o tradisce una Porzia sentimentale. Vi potrebbero addirittura essere interpolazioni tra questi diversi episodi, come tenteremo di dimostrare a proposito del quadro della Sirani.

Se nel primo episodio Porzia sembrava sfidare virilmente il marito, apparirà nei successivi una sposa afflittissima prima dalla sua partenza in esilio, poi dalla morte.

L'esame attento del quadro, nonché il confronto con gli scarsi esempi di quadri sullo stesso tema consentiranno di dimostrare non solo l'originalità del trattamento siraniano ma la complessità di esso, giacché la Sirani potrebbe aver dipinto non una storia, bensi due in questo suo quadro, corrispondenti a due passi della Vita plutarchiana di Bruto. Cercheremo di sostenere l'ipotesi che nel quadretto in secondo piano, la Sirani abbia rappresentato non tanto le ancelle allontanate, di cui si 
parla esplicitamente nella fonte plutarchiana - «e cacciando di camera tutte le sue cameriere» - che non cuciono affatto; punto fondamentale, ma abbia alluso ad un episodio successivo della Vita di Porzia ove ci sono, invece, delle donne che cuciono.

Nel capitolo in cui Bruto, dopo l'attentato, deve esiliarsi Porzia appare molto diversa dalla mulier virilis e rivendicativa dell'episodio precedente: una donna vulnerabile che in stridente contrasto con l'altro episodio, non riesce a nascondere il proprio dolore e viene addirittura tradita da un quadro.

In questo luogo essendo Porcia per tornare a Roma, tentò di nascondere al marito i dolori, che per ciò l'affliggevano, ma la magnanima, e generosa donna fu scoperta da una certa pittura. Il suggetto di questa pittura era una historia Greca, cioè Hettore, che usciva di Troia, accompagnato da Andromacha; laquale haveva preso il figliuolo dal marito, e gli teneva gli occhi addosso. Veggendo Porcia questa pittura, per la somiglianza della passione si diede a piangere, e molte volte appressandosi quivi, sospirò, e pianse amarissimamente. [...]

Sorridendo Bruto, \& io, disse, [non] posso dire hora i versi d'Ettore a Porcia.

A te convien pensare a' tuoi filati,

A le tue lane, e commandar le serve. ${ }^{60}$

Un quadro viene messo in scena e en abyme, giocando un ruolo decisivo nel racconto. L'aneddoto è una perla per chi si dilettasse di pittura e sembra improbabile che la Sirani non l'abbia colto.

L'operazione attesterebbe il grado di raffinatezza ${ }^{61}$ raggiunto dalla Sirani, non certo perché raffigurerebbe nello stesso quadro simultaneamente due episodi che si sarebbero svolti successivamente (espediente comune anche nella pittura primitiva) ma perché avrebbe scelto un brano della vita plutarchiana di Porzia meta-critico: un brano che metteva in scena un quadro e decantava l'espressività della pittura. Quanto non disse Porzia, lo disse il quadro raffigurante gli addii di Ettore ed Andromaca, alla stregua del gesto cui la pur intelligentissima, colta ed eloquente Porzia aveva preferito affidare il suo messaggio. Commenta Du Bosc:

Brutus loue Porcia de ce qu'elle n'est pas ignorante comme Thelemaque. Et en effet elle estoit fort sçavanta \& en Rhetorique, \& en Poësie, \& en Histoire, \& surtout en la philosophie des Stoïciens. ${ }^{62}$ 
Si potrebbe sostenere che il ritratto siraniano di Porzia sia quindi un'opera sul linguaggio e sul modo in cui il gesto e l'immagine possano sostituire e vincere la parola. Opera polemica e «dotta» come non a caso chiamavano Elisabetta gli ammiratori delle raccolte picinardesche, pienamente intonata con la consapevolezza artistica e professionale raggiunta dalla Sirani, secondo la quale la poesia muta sarebbe superiore alla pittura loquace, tanto per esprimersi nel linguaggio di Picinardi. La Sirani rompe con la parità tra pictura et poesis, ovviamente sostenuta da Picinardi e colleghi, per rivendicare la superiorità della poesia muta: quella dei gesti, delle immagini, dei quadri.

Con questo quadro, non avrebbe solo voluto dire qualcosa sulle donne pittrici e su di sé, come ha arguito in particolare Patricia Benahou Philippy, ${ }^{6}$ ma qualcosa sul linguaggio, il che non esclude affatto una lettura in chiave autoritrattistica. Elisabetta si autoritrae da Porzia; una donna che dipinge col proprio sangue, che incide colle forbici, la cui arma potrebbe metaforicamente raffigurare il pennello.

L'episodio sembra segnare un'inversione delle parti rispetto a quello precedente in cui Bruto era stato pris de court e aveva riconosciuto la propria inferiorità rispetto alla moglie come esito della lectio impartita dalla moglie, sia pur con una pedagogia d'urto.

Nell'episodio successivo è Porzia che viene "scoperta" ed è Bruto che, nel contrapporla ad Andromaca, come quella che ha dimostrato di non somigliarle, la richiama all'ordine, invocando la donna greca come contromodello femminile, cui sembrava che Porzia stesse per indulgere o cedere. Questa Porzia sentimentale, lacrimosa, "fusionale" nel senso che non vorrebbe lasciare il marito, viene riportata al livello eroico dell'Anti-Andromaca cui si era issata nel periodo della congiura.

Non potrebbe essere che la Sirani abbia raffigurato in alto e a sinistra Andromaca con le serve? L'Andromaca cosi come "funziona" nel racconto plutarchiano? Cosi come Plutarco avrebbe citato antifrasticamente $i$ versi dell'Iliade, la Sirani avrebbe incastonato nel ritratto di Porzia quello contrappuntistico di Andromaca.

Sembra proprio che la risposta di Bruto alluda all'episodio precedente dell'automutilazione e faccia capire a Porzia di aver "assimilato" la lezione. Sembra quasi che ironicamente la rimandi a questa sua forza esibita nel famoso episodio e al fatto che essa non possa fare a turno o a comodo le parti di Porzia e di Andromaca. In questo episodio successivo, 
a controllarsi e a padroneggiare la situazione è Bruto, a subirla e a tradirsi è Porzia.

\section{Una «bouche très éloquente»}

A sostegno dell'ipotesi di una mise en abyme dell'episodio del quadro di Ettore nonché del collegamento di tale episodio con quello dell' automutilazione ma anche di una chiave di lettura prevalentemente metacritica dell'insieme, addurremo una fonte francese e anteriore al quadro della Sirani: La Femme hérö̈que di Jacques Du Bosc.

Le pagine di Du Bosc presentano il considerevole interesse di soffermarsi a lungo sull'episodio dell'automutilazione riqualificato come: "Etrange précaution de Porcia, avant que d'oser demander le secret de son Mary» ${ }^{64}$ e di offrirci una ricezione seicentesca di quel gesto, tutt'altro che di facile lettura, che contrasta col clamoroso silenzio italiano.

Non solo Du Bosc non ha 'paura' del gesto ammirevole di Porzia, cui dedica una parte specifica di un argomentato elogio di Porzia, tutto imbastito sul confronto con un Bruto decisamente non all'altezza, «en plusieurs choses elle n'esgalle pas surtout Brutus, elle le surpasse»,, "5 ma collega l'automutilazione all'aneddoto del quadro, in quanto varianti complementari di un'idiosincrasia linguistica di Porzia: una eloquenza alternativa, affidata al gesto o all'immagine. La ferita viene definita «une bouche très éloquente»:

ce fut par un langage muet, mais pourtant fort éloquent [...] Parce que s'estant blessée à la cuisse comme nous avons desia dit, sa blessure fut une bouche très éloquente qui descouvrit beaucoup de choses à Brutus: si peu qu'il voulut prendre garde à cette façon de parler, il n'y a point de doute que ce fut son dessein de luy faire sentir adroitement qu'il entreprenoit un dessein fascheux, \& qui peut-estre les obligeroit tous deux à mourir comme Caton, \& qu'elle s'essayoit de bonne heure. ${ }^{66}$

In quanto al quadro l'avrebbe fatto fare e dipingere appositamente per dire a Bruto quanto non poteva esprimere con la bocca:

Et il me semble que cette conjecture est fort raisonnable, puis que c'estoit la façon de parler de Porcia, qui estoit fort discrète \& qui ne s'exprimoit que de la sorte à cause de sa grande modestie \& du grand 
respect qu'elle avoit pour Brutus a qui sans doute elle exprima son regret comme son amour: parce que quand il s'en alla a Athènes, nostre Heroïne pour luy tesmoigner le regret de son absence, fit faire un tableau tiré de l'Histoire de Thelemaque, qui disoit à Dieu à son mary Hector en pleurant alors qu'il alla a la guerre. Ne pouvant luy exprimer de bouche ses ressentiments, comme elle estoit discrète \& généreuse, elle luy exprima par un tableau qu'elle fit faire où elle fit peindre Thélemaque qui accompagnoit son mary Hector, lors qu'il sortoit de ville de Troye pour aller a la guerre; mais en sorte que Thélemaque avait toujours les yeux fichez sur luy en ce départ. ${ }^{67}$

Un'invenzione di Du Bosc giacché nel testo plutarchiano gli occhi di Porzia cadono casualmente su questo quadro. Tra l'altro, c'è un'altra approssimazione: la confusione nominale tra Andromaca e Thelemaque, che documenterebbe una riscrittura libera del passo. Ciò non toglie che l'approfondito commento di Du Bosc sia all'automutilazione che al quadro degli addii di Ettore ed Andromaca costituiscono una favolosa ekfrasis al quadro della Sirani e avallano la nostra proposta non solo di riavicinare questi due passi della vita di Plutarco, ma di pretendere che siano stati entrambi illustrati nel primo e secondo piano del quadro, in quanto emblematici di «la façon de parler» di Porzia, per dirla con Du Bosc.

Questa esegesi francese seicentesca della ferita di Porzia, in termini di bouche éloquente, consente di leggere la Porzia siraniana come un quadro metacritico, portatore di un elogio polemico della pictura, lingua del gesto e dell'immagine, a spese della poesis. Il quadro andrebbe pertanto letto alla stregua di un autoritratto allegorico; un autoelogio da parte di un'artista ormai consapevole della proprio professionalità, come diversamente attestato dal "fenomeno della firma" e dalla fama raggiunta dalla pittrice eroina.

\section{DAL CUCIRE AL DIPINGERE}

Il confronto tra $i$ diversi frammenti della vita plutarchiana di Porzia consente di mettere a fuoco un ritratto più complesso di Porzia che potrebbe corrispondere alle diverse "anime" della donna e-forse? della stessa Sirani: in alcuni casi mulier virilis, in altri, mulier fidelis, in altri ancora, la donna angosciata che sviene, affidando in 
fondo sempre al corpo la fenomenologia - quasi isterica in questo casodel suo dolore, in fine la suicida che in Valerio Massimo conciliava virilità (del mezzo) e coniugalità (del fine), originalità formale e conformismo contenutistico.

Una lettura psicologicamente più sottile potrebbe quindi sostenere che $i$ rapporti tra $i$ due poli del quadro: quello collettivo, tradizionale, scontato; quello eccezionale, eroico, solipsistico, non necessariamente formano i corni di un dilemma. Si pensi al détournement del telaio operato da Penelope nell'Odissea; opera per altro presente sia nella produzione del padre che della figlia Sirani. ${ }^{68}$

A questo punto, la Sirani avrebbe raffigurato due immagini della femminilità, una conforme, l'altra fuori norma, ma non necessariamente incompatibili, così come la Porzia plutarchiana è insieme la donna che ordisce una magistrale congiura solitaria contro il marito e quella che deplora la sua partenza e viene suo malgrado tradita da un'immagine. Se Porzia era tutte e due, anche la Sirani potrebbe riconoscersi sia in quell'operoso collettivo femminile che nell'eroica solitudine di Porzia, in quell'immagine sia tradizionale che trasgressiva.

Dell'operosità femminile, la Sirani avrà avuto ampia esperienza personale, familiare e professionale al punto da non escludere che il quartetto del quadro rappresentasse le sorelle Sirani con la madre; una figura a sinistra, evidentemente più anziana in piedi con indice tassativo diretto verso una cesta di lavoro, sembra rimbrottare le giovani. Giovan Andrea Sirani non aveva forse raffigurato le tre figlie sotto parvenze allegoriche in una tela tuttora a Bologna? ${ }^{69}$ Ovviamente la tesi è un po' azzardata: implicherebbe uno sdoppiamento della stessa Elisabetta, presente sia nel quartetto che nella parte di Porzia in coerenza però con quanto il più fidato biografo, Malvasia, scrisse del carattere servizievole e della sua partecipazione alle più umili mansioni domestiche ${ }^{70}$, tra cui non potevano mancare $i$ cosidetti ouvrages de dames.

Parecchia della poesia encomiastico-pubblicistica, edita da Picinardi ricama proprio sul cucire come metafora del dipingere giocando sulla doppiezza di materie: $:^{7 \mathrm{I}}$ tele, lini... che potevano rientrare sia nel campo del cucito che della pittura. Lo sdoppiamento femminile messo in scena dalla Sirani potrebbe pertanto non ridursi ad una manichea contrapposizione tra Andromaca e Porzia, ma documentare al contrario la profonda ambivalenza di una donna che pur ambendo alla virilità e al riconoscimento professionale, non avrebbe neppure rinunciato alla pro- 
pria femminilità; un teorema, tutto sommato, in linea con la biografia rassicurante di Malvasia che tenne a dipingere la Sirani più come angelo del focolare che virago.

Tra le poesie che si possono considerare una preziosa raccolta di luoghi comuni sulla ricezione della pittrice da parte dei contemporanei, il cucito svolge le due parti che abbiamo individuate: quella di contraltare all'arte della pittrice amazzone

Fui Donna in Terra, e non conobbi Amore;

Sdegnai d'Aracne il trionfar con l'ago,

E per render di gloria il desir pago,

Sol d'Apollo trattai Cetra, e colore. ${ }^{72}$

Il cucire, occupazione consueta, banale, viene assimilato ad un'altra consuetudine femminile: l'amore, come a dire che la pittura implicherebbe una rinuncia sia ad una vita privata che ad una vita 'comune'. Ma il cucito può anche fungere in questa produrione assai ripetitiva, in cui gli autori sembrano a volte ricamare sulla stessa metafora come se qualcuno avesse dato loro il là, da metafora della pittura, ${ }^{73}$ con il duplice vantaggio di conciliare l'arte atipica con un'attività tradizionale:

Sovra lini eruditi Arte sublime

Del Tespio amante i molti stami indora...

Donna, a cui diè Natura idee superne,

Con pinta fila hà le tue glorie ordite? ${ }^{74}$

Per altro, un quadro della Sirani, l'Ercole filante, ${ }^{75}$ purtroppo scomparso, e di grande successo, almeno stando al numero di componimenti poetici scritti in occasione della sua esposizione «nella galleria del Sig. Cesare Leopardi» si prestò a metaforici equivoci di questo genere. ${ }^{76}$ In sostanza, chi scriveva spiegava che Ercole non avrebbe dovuto sentirsi umiliato di quel cucire giacché sia il Tempo filava le ore che l'autore filava ed intesseva corona alla «dotta» Sirani.

Un'altra metafora ricorrente nelle poesie delle raccolte picinardesche, entrambe pubblicate dopo la morte di Elisabetta e comprendenti versi che trattano della pittrice morta o di opere sue, è per l'appunto l'identificazione con le Parche. ${ }^{77}$ Un'identificazione che verrebbe spontanea alla vista del gruppetto di donne filanti, se non che le donne sono quattro 
e non tre. Ciò non toglie che un bel sonetto del Sig. Co. Agostino Berò assimilava la sorte ad un tratto di pittura e la Sirani morta ad una delle Parche, ${ }^{78}$ il che conferisce al cucire/dipingere una dimensione iniziatica di tutt'altra portata rispetto al topos casalingo.

Come la Parca, la pittrice consentirebbe di accedere all'immortalità. La Parca diventa una metafora mitologica ma anche tessile della pittrice, collocata oltre la vita e la morte, in quanto capace di dar vita ed immortalità tramite le opere d'arte. ${ }^{79}$ La figura della Parca consente di appropriarsi della Sirani morta ed eufemizzare la morte cosi come l'immagine della sirena nell'apparato funebre, banalizzata dalle ovvie assonanze col cognome di Elisabetta, potrebbe illustrare l'ambivalenza sociopsicologica della pittrice eroina, del «Mostro dell'Arte».

\section{UN AUTORITRATTO MASCHERATO?}

Per quanto discutibile e anche fastidiosa per certi riguardi, la tentazione di leggere il ritratto di Porzia come un autoritratto è irresistibile. Discutibile dal momento che la Sirani si è autoritratta in modo dichiarato sia in autoritratti "professionali" da pittrice, armata di pennelli e tavolozze, sia in ritratti allegorici (l'Allegoria della Musica I659) o travestiti (la Santina a lato del Battesimo della Certosa), da lei stessa esplicitamente identificata nella Nota come autoritratto. Perché mai cercare nella Porzia un autoritratto se non fu né concepito né rivendicato come tale? Ipotesi fastidiosa, perché nel compiere questa operazione, pare quasi di cadere nella trappola tesa da Vasari col precedente lourd de conséquences del bassorilievo di Properzia de' Rossi, letto in chiave autobiografica; consacrazione storiografica di un duraturo pregiudizio in base al quale le donne non saprebbero trattare di altro che di sé e peggio ancora di un sé sentimentale e/o sessuale. ${ }^{80}$

A contrario, però, si potrebbe sostenere che mentre l'autoritratto consegna un ritratto costruito ed "ufficiale" di sé, un autoritratto involontario o mascherato consentirebbe per l'appunto di avancer masqué. ${ }^{81} \mathrm{Nel}$ caso della Porzia siraniana, la si potrebbe considerare sia un autoritratto ideale (in quanto inno alla virtù femminile) che un autoritratto inconscio, nel quale le sarebbero sfuggite tante cose, non ultimo l'erotismo a dir poco conturbante di questa donna bellissima, che, seduta a gambe larghe, si ferisce con un'espressione alquanto inappropriata? Il quadro si presterebbe alle letture più arrischiate, a qualche indagine 
psicocritica, ma anche alle letture più sagge ed ortodosse. Quella che, in fondo, abbiamo scelta, sostenendo la tesi di un autoritratto professionale: un inno all'eloquenza muta della pittura in perfetta concordanza tematica col titolo della seconda raccolta picinardesca ma anche di un topos culturale: il famigerato confronto tra pictura $e$ poesis. Il confronto non venne trattato però né pacificamente né convenzionalmente dalla Sirani che con la Porzia sbandiererebbe un quadromanifesto a favore della superiorità comunicativa della pictura.

Nessuna delle ipotesi esclude l'altra e il fascino del quadro risiede proprio in quel nodo di letture possibili.

Ci si chiede come mai una donna nubile, chiusa nella casa paterna, descritta come casta e «frigida», ${ }^{82}$ senza nessuna esperienza né del matrimonio né dell'eros, abbia potuto scegliere nella Vita di Bruto un passo cosi scabroso; un passo che trattasse della coppia, dei rapporti tra uomini e donne; un amore che portasse una donna a compiere un gesto cosi stravagante, complicato, autolesionistico?

Sia gli autori di Picinardi che lo stesso Malvasia avevano insistito sul celibato convinto di Elisabetta. La vergine venne 'accoppiata' col padre ${ }^{83}$ nel titolo stesso della Vita malvasiana oppure sposata postumamente con Reni, mentre l'opera veniva configurata come una partenogenesi:

Del tuo fior Virginal lieta e contenta Vanne, o di saggio Padre egregia figlia Senza imenei feconda in te diventa Se tante parti in un sol parto ei figlia. ${ }^{84}$

C’è da stupirsi e può essere che si siano stupiti la stessa Sirani e il comittente che non esposero questa opera, rimasta quasi clandestina, fino alla riscoperta su tutt'altre sponde, in tutt'altro secolo, molto più attrezzato per gestirlo, magari, anacronisticamente.

Se si cercasse però di mettere a fuoco quanto la Sirani avrebbe potuto larvatamente denunciare e/ o rivendicare in questo quadro, non sarebbe abusivo invocare il senso di claustrofobia sprigionato da questa camera; un ambiente opprimente dal quale sia Porzia che Elisabetta pretesero uscire per vie più o meno traverse.

Se la ferita serve a Porzía a dimostrare la sua capacità ad uscire dai confini e dalle mansioni domestiche cui l'avrebbero relegata i pregiudizi di Bruto, il pennello impugnato dalla Sirani sembra svolgere rigorosa- 
mente lo stesso ruolo: dimostrare la capacità ad uscire di casa, come difatti le opere esposte, poi disperse agli angoli dell'Italia ma anche dell'Europa ${ }^{85}$ dimostreranno. In questo gioco delle parti, l'alter ego siraniano di Bruto, quello che nel cosmo siraniano potrebbe fare le veci del referente maschile, sarebbe Giovan Andrea, reo di una "chiusura" attestata da fonti pur reticenti ed inclini a blandire il Sirani padre.

Difatti, il desiderio di uscire, di viaggiare, di spostarsi, sia pur per motivi squisitamente artistico-professionali è uno dei pochi che, pare, siano filtrati dalle pagine delle fonti seicentesche. Lo scrisse Picinardi nell'Orazione, ${ }^{86}$ lo confermò Malvasia nelle note inedite, ${ }^{87}$ depositarie di tutto quanto Malvasia non volle nè poté dire nella Felsina. ${ }^{88}$ Il quadro potrebbe raffigurare la situazione di chiusura/clausura più o meno letterale nella quale venivano costrette le donne, mentre il sangue di Porzia potrebbe raffigurare quello stesso «sudore e sangue» che secondo Picinardi, costò alla Sirani l'acquisizione di un sesso virile. ${ }^{89}$ «Sudore e sangue» non solo in senso traslato, se si pensi alla malattia che l'avrebbe portata via un anno dopo.

Uno dei portati del quadro sta proprio nella messa in scena di quella capacità femminile di ritagliarsi uno spazio di libertà nel carcere domestico. Tragicamente, è quanto poi avvenne in quell'episodio finale della vita plutarchiana di Porzia, in cui essa riusci nonostante i provvedimenti presi per precluderle il suicidio, ad evadere da queste costrizioni, ricorrendo ad uno stratagemma - la conversione dei carboni, fonte di calore, in un veleno mortifero - cosi come la Porzia automutilante aveva convertito forbici o rasoio in pugnale.

Frédérique Dubard de Gaillarbois 
i. Carlo Cesare Malvasia, Felsina pittrice Vite dei pittori bolognesi, Bologna, per l'erede di Domenico Barbieri, i678, p. 473.

2. Proprietà dell'Herbert Johnson Museum di Ithaca, venne poi acquisito dalla Stephen Warren Miles and Marylin Ross Miles Foundation (Houston). Si trova ormai al palazzo Fava, sede della Fondazione Carisbo che l'ha acquistato e «rimpatriato» nel 2008 .

3. Elisabetta Sirani pittrice eroina I638-1665, a cura di J. Bentini \& V. Fortunati, Bologna, 2004. Il catalogo della Mostra fornisce un utile état des lieux degli studi siraniani, una rassegna della ricezione postuma della Sirani; campo suscettibile di integrazioni ed aggiornamenti in particolare in ambito extra-italiano. Cfr. E. Lehours, Elisabetta Sirani: de l'amazone à la sirène, tesi di dottorato discussa a Paris-IV, il ıо/ı 2/2010.

4. Né le curatrici della Mostra del 2004 né la monografia di A. Modesti, pubblicata lo stesso anno, hanno indugiato sulla Porzia, riprodotta male e sbrigativamente commentata, cfr. A. Modesti, Elisabetta Sirani una virtuosa del Seicento bolognese, Bologna, Editrice Compositore, 2004. Si trovano spunti in B. Bohn, F. Frisoni, A. Modesti, W. Chadwick. Cfr. la nota stilata da Irene Graziani in Elisabetta Sirani pittrice eroina I638-1665 cit., p. 2 17. Più ambiziose le pagine dedicate da P. Benahou Philippy all'interpretazione del quadro. Cfr. P. Benahou Philippy, Painting women: cosmetics, canvases, and early modern culture, 2006. Segnaliamo i nostri contributi: F. Dubard de Gaillarbois, Elisabetta Sirani, Malvasia, Porzia: de la «morte violenta» à la «pittura che violenta», in Art et violence dans le vies d'artistes. Colloque international, Italie, France, Angleterre xvi ${ }^{\mathrm{e}}$-XvinI ${ }^{\mathrm{e}}$ siècles, Paris 9-I I / I 2 / 20 Io, Paris, Éditions Jonquières, 201 2, pp. 57-84; F. Dubard de Gaillarbois, Elisabetta Sirani's Porcia wounding her thigh (1664). A piece mute éloquence or a meta-painting?, in «Icon. Journal of Iconographic Studies», 7 (2013), pp. $235-48$.

5. Cfr. W. Chadwick, Women, Art and Society, Thames and Hudson, I997, pp. Iо I-I03.

6. A. Sutherland Harris, Le grandi pittrici (I550-1950), catalogo della mostra, a c. di A. Sutherland Harris, L. Nochlin, Milano, Feltrinelli, pp. I 5-42, pp. I47-50; F. Frisoni, Elisabetta Sirani, in La scuola di Guido Reni, a c. di E. Negro, M. Pirondini, Modena, Artioli, I992, pp. 345-47.

7. I. Graziani, Il cenacolo di Elisabetta Sirani, in Elisabetta Sirani pittrice eroina I638-1665 cit., pp. I I9-35.

8. B. Bohn, Il fenomeno della firma: Elisabetta Sirani e le firme dei pittori a Bologna, in Elisabetta Sirani pittrice eroina 1638-1665 cit., pp. 107-1 8.

9. Malvasia, Felsina pittrice, p. 473. Cfr. nota 25 infra.

io. Giovan Luigi Picinardi, Ilpennello lagrimato Orazione funebre del signor Gio. Luigi Picinardi con alcune poesie in morte della signora Elisabetta Siranipittrice famosissima, in Bologna, per Giacomo Monti, I66; ; Giovan Luigi Picinardi, La poesia muta celebrata dalla pittura loquace. Applausi di nobili ingegni al pennello immortale della S.ra Elisabetta Sirani pittrice bolognese, per gli heredi di Evangelista Dozza, Bologna, I 666. 
i i. Malvasia, Felsina pittrice, p. 473.

I 2. F. Dubard de Gaillarbois, Vasari selon Malvasia on il tirannico giogo dell 'ipse dixit', in La réception des Vite de Giorgio Vasari en Europe (XVI-XVIII siècle). Colloque international, Paris, INHA, Paris I, Paris III, 27-28-29 octobre 20I I, à paraittre aux éditions Droz.

i 3. Malvasia, Felsina pittrice, IV, p. 399.

i4. Carlo Cesare Malvasia, Vite di Pittori Bolognesi (Appunti inediti), ed. A. Arfelli, Bologna, Commissione per i testi di lingua I961, p. 95.

I 5. W. Chadwick, Women, Art, and Society, London, Thames and Hudson, I994, p. IOI.

I6. R. Morselli, Collezionisti e quadrerie nella Bologna del Seicento, Documents for the history of collecting: italian inventories, Getty Trust Publications, Los Angeles, I998, pp. 4I6-2 I. La Morselli attesta un rapporto tra i Tassi, ex-nobili «caduti in disgrazia» e i Gessi, membri a tutti gli effetti dei Gelati e sottolinea lo snobismo di Malvasia che avrebbe silenziato queste collezioni da parvenus.

17. Cfr. G. Perini, Strutture e funzione delle mostre d'arte a Bologna nel sei e settecento, in «Accademia Clementina. Atti e memorie», vol. 26 (1990), pp. 293-340; A. Modesti, Patrons as agents and Artists as Dealers in Seicento Bologna, in The Art Market in Italy: Isth - I7th centuries, ed. M. Fantoni, L. Matthew, S. MatthewsGrieco, Modena, Cosimo Panini Editore, 2003, pp. 367-88.

I 8. Dispiace non poter vedere questo quadro scomparso, il cui soggetto non è certo originale come la Porzia e anche la Timoclea, opere più tardive, ma presenta indubbia affinità tematiche sul tema dell'inversione delle parti e dei ruoli tra maschile e femminile. Interessante la dicitura della voce siraniana nella Nota delle pitture: «Un'altra testa similmente d'una Iole per l'istesso illustrissimo sig. Berlingiero Gessi, che la mandò a donare al sig. Cesare Leopardi, Cavalliere della città d'Osimo, ond'io cangiai la pelle di Leone alla detta Iole, e glie la formai più tosto di Leopardo", cfr. Malvasia, Felsina pittrice, p. 47I, ove la pittrice si premura di sottolineare la propria trovata encomiastico-maliziosa per ammiccare al cognome del destinatario dell'opera e documenta l'uso di ritocchi di convenienza in funzione del destinatario.

I9. Ai due nomi fatti da Bohn (cfr. Bohn, The antique heroines of Elisabetta Sirani cit., pp. 52-79): Ercole de'Roberti (prima), Nicolas-Bernard Lepicié (dopo), aggiungiamo quelli di A. Tiarini e F. Solimena.

20. Ad esempio, Jacopo del Sellaio, Fra Bartolomeo, F. Primaticcio, Nicolas Prévost, Charles Mellun, Pierre Regnier, Luca Giordano, José de Ribera, Giuseppe Ghezzi.

2 I. L'Indice della Felsina indicava altre due Porzia, una di Guido Reni «ora presso li sig. Rossi e Fabri» (Malvasia, Felsina pittrice, II 63), oggi a Genova, collezione Durazzo Pallavicini (I625-26), una di Savonanzi (Malvasia, Felsina pittrice, II 304 ). 
22. Quella di Savonanzi era a sua volta tributaria di Reni, cfr. «L'avvenenza dei volti, che accompagnava sempre le opere uscite dal pennello di Guido, fu presa ad esempio da Emilio, allorché ebbe a dipingere per Monsignor Marazzani vescovo di Senigallia la Circe, la Porzia, l'Artemisia, e l'Arianna: quadri che di presente trovansi in un palazzotto di villa di questi signori», A. RiccI, Memorie storiche delle arti e degli artisti della Marca di Ancona, vol. I, p. 24I.

23. Un quadro intitolato Porzia e Catone è stato venduto dalla casa d'aste Finarte, (lotto 76), il I 9-I I-1997. Sebbene attribuito alla «scuola» di Tiarini, non può non interessarci in quanto raffigura una Porzia sdraiata che mostra il piede o una fascia insanguinata mentre 'Catone' fa un gesto di stupore. Che sia precedente o contemporaneo al nostro, autentico o meno, il trattamento risulta due volte tradizionale rispetto a quello siraniano e per la presenza di Bruto e per il pudico spostamento dalla coscia al piede. Tra l'altro, il titolo sarebbe sbagliato: si tratterebbe non di «Porzia e Catone», ma ovviamente di «Porzia e Bruto».

\section{Malvasia, Felsina pittrice, p. 473.}

25. Robert Garnier, Porcie, tragédie françoise, représentant la cruelle et sanglante saison des guerres civiles de Rome, propre et convenable poury voir depeinct la calamité de ce temps...Paris, Robert Estienne, i 568; Guyon Guerin de Bouscal, La Mort de Brute et de Porcie on la Vengeance de la mort de Cesar, Paris, T. Quinet, 1635; Claude Boyer, La Porcie romaine: tragédie par l'abbé C. Boyer, Paris, A. Combé, I646.

26. Sebbene le nostre ricerche di Porzie automutilanti nelle letteratura italiana del '500 e del ' 600 siano risultate deludenti, non sono state abbastanza approfondite per escludere con certezza la presenza di Porzia sia nel teatro che nel melodramma italiano. Da notare che Shakespeare nel suo Giulio Cesare rappresentato

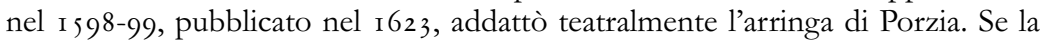
ferita alla coscia non viene censurata, non viene messa in scena: «Tell me your counsels, I will not disclose 'em: / I have made strong proof of my constancy, / Giving myself a voluntary wound / Here in the thigh: Can I bear that with patience, / And not my husband's secrets?», William Shakespeare, Giulio Cesare, a c. e trad. di F. Giacomantonio, Roma, Newton, 2004, (II, I), vv. 292-3 I 5 , p. 66.

27. La vedova suicida appare come una personificazione della repubblica sconfitta in Guerin de Bouscal. Genericamente, le guerre di religione nel 'soo, la Fronde e le reggenze nel ' 600 consentono un'attualizzazione e un'acclimatation francese di vicende storiche non più intonate coll'Italia seicentesca.

28. «Seconde instruction pour les Marys [...] que les Femmes leur sont données pour Assistantes \& pour Cooperatrices: qu'elles doivent avoir leurs places dans le cabinet aussi bien qu'au Lict; \& leur part aux affaires non moins qu'à la table», Pierre Le Moyne, La Gallerie des femmes fortes, Paris, par la compagnie des marchands libraires du Palais, I665, p. 290; vera e propria parafrasi della «concione» plutarchiana di Porzia.

29. «En plusieurs choses elle n'esgalle pas surtout Brutus, elle le surpasse», Jacques Du Bosc, La Femme héroïque ou les héroïnes comparées avec les héros en toutes sortes de vertus par le P. Du Bosc et Plusieurs Reflexions Morales a la Fin de Chaque 
Comparaison, Paris, Antoine de Sommaville et Augustin Courbe, I645, 2 vol., p. 397.

30. Principale appiglio ad un interesse dei Sirani per la politica, sarebbe la presenza nella loro biblioteca del Tarquinio di Virgilio Malvezzi, noto trattato sulla tirannia, presentato dall'autore come una «Tragedia utile», anche se la presenza di storici moderni (Giovio) dimostra l'apertura intellettuale dei Sirani, la cui biblioteca era ben lungi dal limitarsi ad interessi professionali. Cfr. S. Sabbatini, Per una storia delle donne pittrici bolognesi: Anna Maria Sirani e Ginevra Cantofoli, in «Schede umanistiche», (1995), pp. 85-гог.

3r. Du Bosc, La Femme héröque, p. 4i4: «Que si le mépris de la mort est le principal fruit de la philosophie, il me semble que Porcia sut philosopher encore plus noblement que Brutus».

32. Il suicidio vantava illustri credenziali filosofiche (Socrate, Seneca tanto per farne due nomi, quest'ultimo godendo per altro di una fortuna iconografica cui sarebbe potuta esser sensibile la Sirani). Nei casi di Catone, Porzia e Bruto i moventi filosofici e politici si potevano sovrapporre.

33. A Porzia venne attribuita da diverse fonti, Plutarco per primo, la padronanza della cultura filosofica, Du Bosc, La femme héroïque, p. 427: «Et en effet elle estoit fort sçavante \& en Rhétorique, \& en Poësie, \& en Histoire, \& surtout en la philosophie des Stoïciens». Compare sia pur lapidariamente nel dizionario delle donne filosofe di Gilles Ménage, sebbene la sbrigherà con una piroetta: «Porcia. Fille de Caton, femme de Brutus. Plutarque dans son Brutus, la nomme philosophe. Son histoire est trop connue pour que je la rapporte ici», Gilles Ménage, Histoire des femmes philosophes (i 69o), p. 77. Infine Pierre Le Moyne, La Gallerie des femmes fortes, p. 278: «Cette peinture est d'une Magnanime, qui meurt d'affliction \& d'amour; \& meurt constamment et en stoïque. C'est la célèbre Porcie, qui fut rivale d'un Pere Defenseur de la liberté, et d'un Mari exterminateur de la Tyrannie: et qui renouvelle au siècle du luxe et des délices, la Vertu \& la sévérité de la primitive Republique et eut la Vertu en héritage et la philosophie en douaire».

34. La spiegazione di uno scarto così notevole tra l'approccio francese e italiano di Porzia nel seicento meriterebbe uno studio approfondito. Ci limiteremo ad osservare che la "fiaccola" filogina così vivace nell'Italia cinquecentesca sembra migrare nella Francia seicentesca e risentire fortemente delle varie reggenze francesi (Caterina de' Medici, Maria de' Medici, Anne d'Autriche) come leva politica a quella voga delle femmes fortes, cfr. I. Maclean, Woman Triumphant. Feminism in French Literature 1610-1652, Oxford, Clarendon Press, 1977.

\section{Cfr. Sabbatini, Per una storia delle donne pittrici bolognesi cit., pp. 8 5-Iо I.}

36. Si potrebbe notare che lo stesso avviene ad Alessandro Magno nel quadro di Timoclea (1659) ove è presente la maschilità negativa personificata dal capitano stupratore. Degno di nota anche il fatto che la Sirani intitoli Iole il quadro raffigurante Ercole filante, il che vale anche per Dalila (senza Sansone). Il partner maschile o viene cancellato dal quadro e comunque dal titolo; un'innegabile discontinuità col trattamento tradizionale di queste coppie pittoriche, anche se la Sirani non ne aveva il monopolio. 
37. Malvasia, Felsina pittrice, p. 475: «Un Alessandro Magno quando con violenza vuole dalla Sibilla Delfica gli oracoli sopra la guerra persiana, mezze figure del naturale, con una testa di un paggio dietro al detto Alessandro, per un dilettante di pittura. Un simile di Alessandro, quando col suo sigillo fà cenno di sigillare la bocca ad Efestione dopo avergli letta la lettera scrittagli dalla madre, per il sig. Duca della Mirandola». Fra l'altro, un quadro del I664 come la Porzia, mentre la Timoclea risulta il quadro plutarchiano più precoce in quanto del 1659: «Una Timoclea grande del naturale, gettante il Capitano nel Pozzo, per il signor Andrea Cattalani». Si trova una conferma a posteriori e un'attestazione di quel quadro plutarchiano scomparso nella raccolta La poesia muta che trascrive un sonetto del Sig. Vincenzo Maria Marescalchi intitolato «Dello stesso, per l'Historie di Plutarco»: «Là ch'egli è invitto, e qui Immortal s'avisa / Da una Vergin l'Eroe. / Chi più bell'opra Fè, / Alessandro, la Fata, o pure Elisa?», Picinardi, La poesia muta celebrata dalla pittura loquace, p. 76.

\section{Sabbatini, Per una storia delle donne pittrici bolognesi cit., p. 88.}

39. Vite di Plutarco Cheroneo de gli Huomini illustri greci et romani (L. Domenichi), parte seconda, Venetia, Felice Valgrisio, I 562 , p. 204.

40. Vittorio Alfieri, Bruto secondo (1789) in Tragedie, ed. B. Maier, Torino, Einaudi, 2003: «"Or, mira; donna non sono". E in cosí dir, cadersi / lascia del manto il lembo, e a me discuopre / larga orribile piaga a sommo il fianco. / Quindi soggiunge: "Questa immensa piaga, con questo stil, da questa mano, è fatta, / or son piú giorni: a te taciuta sempre, / e imperturbabilmente sopportata / dal mio cor, benché infermo il corpo giaccia; / degna al fin, s’io non erro, questa piaga / fammi e d'udire, e di tacer, gli arcani / di Bruto mio».

4I. Oltre a Valerio Massimo, vanno ricordate come fonti su Porzia passi di Marziale, Epigrammi (I, 43) e di Appiano, Guerre civili (IV, I 36$)$.

42. Valerius Maximus, Factorum dictorumque memorabilium libri $I X, \mathrm{IV}, 4,6,5$ : «De amore coniugali».

43. Giovanni Boccaccio, De mulieribus claris, Tutte le opere di Giovanni Boccaccio, vol. io (I 967), a c. di V. Branca, LXXXII, p. 328: «De Portia Catonis Uticensis filia».

44. Di cui è ragionevole pensare che i Sirani avrebbero potuto accedere, visto che possiedevano la Genealogia degli dei, cfr. Sabbatini, Per una storia delle donne pittrici bolognesi cit.

45. Boccaccio, De mulieribus claris, p. 389 .

46. Cfr. Giovanni Sabadino degli Arienti, Gynevera de la clare donne, Bologna, Romagnoli dall'Acqua, i 888, p. I70.

47. Cfr. Galeazzo Flavio Capella, Della eccellenza e dignità delle donne, a c. di M. L. Doglio, Roma, Bulzoni, I988, p. 45: «De la dilezione d'amore».

48. Giovanni Boccaccio, Elegia di Madonna Fiammetta, VI, 6: «Vennemi poi nel pensiero li velenosi sughi, li quali per adietro a Socrate, a Sofonisba e Anibale e a 
molti altri prencipi l'ultimo giorno segnarono: e questi assai alli miei piaceri si confecero. Ma veggendo che a cercare d'averli tempo si convenia interporre, e dubitando non in quel mezzo si mutasse il mio proponimento, di cercare altra maniera immaginai. E pensato mi venne di volere intra le ginocchia, come molti già fecero, rendere il tristo spirito: dubitando d'impedimento, ché 'l vedea, ad altra spezie di pensieri trapassai. E questa cagione medesima li accesi carboni di Porzia mi fece lasciare».

49. Vittoria Colonna, Rime de Vittoria Colonna [...], Bergamo, Pietro Lancellotti, I760, Canto II.

50. Cfr. M. Fumaroli, L'école du silence. Le sentiment des images au XVII siècle, Paris, Flammarion, 2008. Stando a Bohn, l'iconografia sacra rappresenterebbe il $42 \%$ della produzione siraniana, quella allegorica, pittura di storia antica o mitologica, il $15 \%$, cfr. Bohn, The antique heroines of Elisabetta Sirani cit., p. 60.

5 I. Malvasia, Felsina pittrice, p. 400: «Alli 3. Gennaro, fu in casa nostra la signora Duchessa di Bransvich, a vedermi dipingere, dove io in sua presenza feci un Amorino d'età d'un anno, significando l'Amor proprio, mostrando volersi ferire da se con una saetta, rimirandosi Nello specchio. Intendami chi può, che m'intend'io ec.».

52. A questo punto, due possono essere le ipotesi: minimalista o impegnativa. Potrebbe trattarsi di un'espressione idiomatica, un'espressione proverbiale intesa a valorizzare la dimensione allegorica dell'opera e il consecutivo sforzo intellettuale richiesto allo spettatore oppure vi sarebbe un riferimento esplicito da parte di Elisabetta al proemio di un celebre canto (Orlando furioso XLIII 4-5), quello della «prova del nappo» cui (saggiamente o amaramente?) rifiuta di sottoporsi Rinaldo. In quel proemio l'Ariosto si sfogava denunciando la scarsa affidabilità e l'avarizia delle donne alludendo ad una sua esperienza personale. Il carattere misogino dello sfogo potrebbe deporre a sfavore del riscontro senonché il verso viene addotto e convertito a motto di un'impresa sull'amor proprio, il cui corpo sarebbe un angelo che si ferisce da sé. Nel qual caso Elisabetta Sirani denuncerebbe la prevalenza dell'amor proprio sull'amor altrui in perfetta linea col disincanto o l'amarezza ariostesca. Interessa questo possibile ammiccamento all'Ariosto in quanto sia i versi ariosteschi che il gesto di Porzia sarebbero accomunati dalla stessa tematica: i rapporti di coppia, che, paradossalmente, sembrano interessare alla nubile o celibe Sirani. Non si tratta neppure di leggere l'automutilazione di Porzia come une denuncia di amor proprio. Semmai era l'amor proprio di Bruto che veniva denunciato con questo gesto. Ma sottolineare quanto la Sirani fosse consapevole delle letture traslate che si potevano fare di un gesto che compare in tre 'generi' diversi: pittura di storia profana, sacra, pittura allegorica. Secondo Manaresi, la Sirani avrebbe alluso al ruolo della dote nelle strategie matrimoniali, di cui lei stessa avrebbe fatto le spese, cfr. A. Manaresi, Elisabetta Sirani la vita, l'arte, la morte, la tradizione del veneficio, il processo, i prodromi, l'autossia, la causa mortis, versi e prose, appendice sulle opere, Bologna: N. Zanichelli, I 898 .

53. Opera che godeva di una fortuna iconografica cui il padre stesso di Elisabetta aveva dato almeno due contributi, Giovan Andrea Sirani, Bradamante aggredisce il 
negomante Atlante (1650-55), Pinacoteca Nazionale di Bologna, Angelica che si sottrae a Ruggiero, Cassa di Risparmio di Cesena (I668). Cfr. L'Arioste et les Arts, M. Paoli, M. Preti-Hamard (dir), Officina Libraria/Editions du Louvre, 20 I 2.

54. Cfr. Francesco Petrarca, Rvf ios, i 7.

55. Guilio Cesare Croce, La Gloria delle donne, i 590.

56. Ad esempio, il convegno Plutarque de l'Âge classique an XIX siècle. Présences, interférences et dynamique, Toulouse, Université de Toulouse-Le Mirail, I 3, I4, I 5 mai 2009 , di cui la Porzia siraniana ha fatto l'icona-manifesto.

57. La Porzia suicida si poteva riallacciare alla serie di belle suicide, di considerevole e conturbante successo nell'Europa seicentesca in generale, bolognese, in particolare: si pensino alle varie Didoni (cui poteva essere subliminalmente riallacciata la Sirani, a volte nominata Elisa nelle poesie pubblicate dal Picinardi, altro nome di Didone) alle Lucrezie, Cleopatre ritratte dai vari Guidi, Cagnacci, Guercino, Sirani padre e figlia.

58. Du Bosc, La Femme héroüque, p. 4i 6: «des le commencement du dessein de son Mary, elle essaye sur sa cuisse, ce que depuis elle eut fait sur son sein, si on ne l'eut contrainte de se servir de charbons au lieu de se servir d'une épée: cette blessure qu'elle se fit fut un gage certain de ce qu'elle se vouloit faire aussi bien que Caton».

59. Scudery, Les femmes illustres ou les harangues hérö̈ques de $M$. de Scudéry avec les Véritables Portraits de ces Heroines, tirez des Medailles Antiques, A Lyon, chez François Comba, I667, p. I 35: «Je fus de la conspiration, puisque je la sceu auparavant qu'elle fut exécutée: il est donc bien iuste que ie suive le destin de Brutus: \& sçachez Volumnius que celle qui eut l'ame assez ferme pour se donner un coup de poignard pour en souffrir la douleur, et la cacher afin de témoigner à son mary qu'elle sçauroit bien celer un secret; ne changera pas aisément la résolution qu'elle a prise de mourir. L'image de Caton \& celle de mon cher Brutus me remplissent tellement l'esprit que ie ne vois plus autre chose».

6o. Vite di Plutarco Cheroneo de gli Huomini illustri greci et romani (L. Domenichi), parte seconda, Venetia, Felice Valgrisio, i 562, XXVII, p. 2 io.

6r. Una raffinatezza meno individuale che epocale stando alle considerazioni di G. Perini per cui i pittori si sarebbero appropriati tecniche letterarie di citazione. Porzia offrirebbe un ottimo esempio di quel sincretismo artistico-letterario e di un citazionismo diretto ed indiretto, cfr. G. Perini, Implicazioni semiotiche nella lettura dei quadri di uno storico, conoscitore e letterato del Seicento, in «Notizie da Palazzo Albani», 2005-2006 [ma 2007], pp. 14I-58.

62. Du Bosc, La Femme hérö̈que, p. 427.

63. Cfr. Benahou Philippy, Painting women: cosmetics, canvases, and early modern culture cit., p. 6o: Porcia è interpretata come «a looking glass for the painting woman». L'eroina «figures the early modern woman painter's journey from privacy to 
publicity», «Her story plays out the imagined moral deformity of the painting woman». Il «self-wounding act» risulta «self-definition», «self-authoring act»; «the painting overtly meditates upon the possibility of women's creative selfauthorship».

64. Du Bosc, La Femme hérö̈que, p. 460.

65. Du Bosc, La Femme hérö̈que, p. 420.

66. Du Bosc, La Femme héroïque, p. 4I I: «on peut voir un échantillon dans cette belle harangue qu'elle fit a Brutus, avant que de luy montrer sa blessure, \& de luy en dire la cause».

67. Du Bosc, La Femme hérö̈que, p. 427.

68. La Nota della Sirani registrava «una Circe» in coppia con «un Ulisse», cfr. Elisabetta Sirani pittrice eroina I638-1665, cit., p. 2 I 2 . Il padre era autore di un Ulisse e Circe, Roma, Pinacoteca Capitolina.

69. Allegoria delle tre arti (Pittura, Musica, Poesia) I663, Pinacoteca Nazionale di Bologna.

70. Picinard, Il pennello lagrimato Orazione funebre del signor Gio. Luigi Picinardi con alcune poesie in morte della signora Elisabetta Sirani pittrice famosissima, p. I 3: «Palesossi d'una umiltà sì profonda, che non sdegnò d'abbassare ben mille volte agli esercizi domestici ancora più vili quell'animo che pure sempre avea fisso all'acquisto della gloria».

7 I. A. Barbieri in Picinard, Il pennello lagrimato Orazione funebre del signor Gio. Luigi Picinardi con alcune poesie in morte della signora Elisabetta Sirani pittrice famosissima, p. 30 : «Tesser à l'occhio inganni, e quasi al tatto».

72. In Picinardi, Il pennello lagrimato Orazione funebre del signor Gio. Luigi Picinardi con alcune poesie in morte della signora Elisabetta Sirani pittrice famosissima, p. I 5.

73. Picinardi, Il pennello lagrimato Orazione funebre del signor Gio. Luigi Picinardi con alcune poesie in morte della signora Elisabetta Sirani pittrice famosissima, p. 53.

74. Picinardi, La poesia muta celebrata dalla pittura loquace, p. 52: «Del Signor C Bocc.».

75. Modesti, Elisabetta Sirani una virtuosa del Seicento bolognese cir., p. 2 I6: «nell'anno I 662 sono annotati ben tre opere [Ercole, Ercole che fila, Iole] raffiguranti Ercole, ma due di questi destinati a Cesare Leopardi e a Berlingiero Gessi sono dipinti, come mi indica Vera Fortunati, per accompagnare una Iole e presentano il semidio in atto di filare, sottomesso al giogo della regina di Lidia che lo tenne per schiavo ed amante».

76. Picinardi, La poesia muta celebrata dalla pittura loquace, p. 50 : «Spettator, che qui miri / Della dotta Sirani alto valore / Non ben conosci in queste tele spresso. / Pensi, ch'io fili, e a lei Corona intesso». 
77. Picinardi, Il pennello lagrimato Orazione funebre del signor Gio. Luigi Picinardi con alcune poesie in morte della signora Elisabetta Sirani pittrice famosissima, p. 23: «Piangano le Parche, che troncandoti lo stame di questa fragile Umanità, ti filarono una vita immortale, essendo il morire glorioso, un vivere eterno».

78. Malvasia, Felsina pittrice, p. 480: «Apri, e vedrai, che ad onta di Natura, Sanno le Parche ancor Pittrici accorte, / Formar de l'human fasto una figura. Apri, e saprai, come la nostra Sorte / Altro non è, che un tratto di pittura, / Che dà colori in vita, ed ombre in morte».

79. V. M. Marescalchi in Picinardi, Il pennello lagrimato Orazione funebre del signor Gio. Luigi Picinardi con alcune poesie in morte della signora Elisabetta Sirani pittrice famosissima, p. 27: «Non è questa colei, che di sue tele / Tela hà sì forte a mille vite ordita».

8०. Cfr. F. Dubard de Gaillarbois, La double Vie de Properzia de'Rossi: d'une biographie d'artiste (I550) à une biographie philogyne (I568), in Nouvelles sources et nouvelles méthodologies de recherche dans les études sur les femmes, Des idées et des femmes, sous la dir. de G. Leduc, Paris, L'Harmattan, 2004, pp. 75-88.

8 I. F. Dubard de Gaillarbois, «Larvata prodeo». Travestissements divers dans les autoportraits des femmes-artistes italiennes à la Renaissance, in Travestissement féminin et liberté(s). Des idées et des femmes, sous la dir. de G. Leduc, Paris, L'Harmattan, 2006, pp. 30I-I 2.

82. Ad esempio Picinardi garantiva dei «costumi, la candidezza de' quali non fu mai annerita dal fumo delle facelle di quello Arciere, ch'ella non conobbe, che sù le tele, onde non le fu di mestieri lavarsi nella fonte di Cupido in Cizico, l'onde della quale sanano dalla febbre amorosa», Malvasia, Felsina pittrice, p. 459.

83. In proposito è interessante notare un'altra coincidenza con Porzia presentata come figlia di Catone e "accoppiata" ora col padre, ora col marito, le cui posizioni e destini finirebbero quasi coll'identificarsi.

84. Picinardi, La poesia muta celebrata dalla pittura loquace, p. 62.

85. Picinardi, Il pennello lagrimato Orazione funebre del signor Gio. Luigi Picinardi con alcune poesie in morte della signora Elisabetta Sirani pittrice famosissima, p. 4: «Piange il Reno di Felsina, e sul di lui nobil margo deploro ancor'io lo scorno della Natura, il prodigio dell'Arte, la gloria del Sesso Donnesco, la Gemma d'Italia, il Sole della Europa, ELISABETTA SIRANI».

86. Picinardi in MAlvasia, Felsina pittrice, p. 6 г 2: «ebbe oltramodo l'animo inclinato a viaggiar fuori delle mura paterne, ma se non le fu concesso di pellegrinare furiera per l'universo delle sue glorie la Fama, stimando menzoniera la sentenza di Tucidide, che asserisce quella donna esser più degna di lode, la cui fama è contenuta fra le pareti de' Lari privati: conosceva ben ella che non s'ha a nascondere la virtù, ma che tutto il mondo dee servire di teatro alle sue geste gloriose».

87. Carlo Cesare Malvasia, Vite di Pittori Bolognesi (Appunti inediti), a c. di A. Arfelli, Bologna, Commissione per i testi di lingua, I96r, p. ıог: «Non desiderò 
cosa maggiormente che di viaggiare e di mutar paese, e suo padre la insperanzava e si dichiarava e prometteva che, aprendosi qualche onorevole occasione di andare in Francia o a Roma, l'avrebbe abbracciata e l'avrebbe sodisfatta».

88. Si ricordi anche un aneddoto riferito dallo stesso che fa toccare con mano gli ostacoli alla crescita artistica recati dalla forzata sedentarietà, in cui la ragazza scopre Correggio tramite una copia fattane da un allievo del padre, Giovan Battista Zani, che, recatosi, lui, a Parma, Malvasia, Vite di Pittori Bolognesi (Appunti inediti), p. ıоo: «anzi, che essendo tornato di Parma, dove era stato a studiare su le cose del Correggio, il Zani, scolare già del detto Signor Giovanni Andrea la copia che avea fatto colà, perché ed egli e la Signora Isabetta vedessero lo studio ed il profitto».

89. Picinardi, Il pennello lagrimato Orazione funebre del signor Gio. Luigi Picinardi con alcune poesie in morte della signora Elisabetta Sirani pittrice famosissima, p. I I. La metafora dell'androginia viene ribadita più avanti, p. I 8: «con destra di Femmina trattò Pennelli Maschili».

Ringraziamo per le gentili e diversamente preziose riletture: Vera Fortunati, Irene Graziani, Ottavia Nicoli, Giovanna Perini. 